\title{
BMJ Open Implementation and use of mHealth home telemonitoring in adults with acute COVID-19 infection: a scoping review protocol
}

\author{
Nurlan Dauletbaev (D , , ${ }^{1,2}$ Sebastian Kuhn, ${ }^{3}$ Svea Holtz, ${ }^{4}$ Susanne Waldmann, ${ }^{5}$ \\ Lukas Niekrenz, ${ }^{6}$ Beate S Müller (i) , ${ }^{4}$ Carla Bellinghausen, ${ }^{7}$ Michael Dreher, ${ }^{6}$ \\ Gernot G U Rohde, ${ }^{7}$ Claus Vogelmeier ${ }^{1}$
}

To cite: Dauletbaev N Kuhn S, Holtz S, et al. Implementation and use of mHealth home telemonitoring in adults with acute COVID-19 infection: a scoping review protocol. BMJ Open 2021;11:e053819. doi:10.1136/ bmjopen-2021-053819

- Prepublication history and additional supplemental material for this paper are available online. To view these files, please visit the journal online (http://dx.doi.org/10.1136/ bmjopen-2021-053819).

Received 24 May 2021 Accepted 06 September 2021

A) Check for updates

(c) Author(s) (or their employer(s)) 2021. Re-use permitted under CC BY-NC. No commercial re-use. See rights and permissions. Published by BMJ.

For numbered affiliations see end of article.

Correspondence to Dr Nurlan Dauletbaev; nurlan.dauletbayev@unimarburg.de

\section{ABSTRACT}

Introduction mHealth refers to digital technologies that, via smartphones, mobile apps and specialised digital sensors, yield real-time assessments of patient's health status. In the context of the COVID-19 pandemic, these technologies enable remote patient monitoring, with the benefit of timely recognition of disease progression to convalescence, deterioration or postacute sequelae. This should enable appropriate medical interventions and facilitate recovery. Various barriers, both at patient and technology levels, have been reported, hindering implementation and use of mHealth telemonitoring. As systematised and synthesised evidence in this area is lacking, we developed this protocol for a scoping review on mHealth home telemonitoring of acute COVID-19. Methods and analysis We compiled a search strategy following the PICO (Population, Intervention, Comparator, Outcome) and PRISMA-ScR (Preferred Reporting Items for Systematic Reviews and Meta-Analyses recommendation for Scoping Reviews) guidelines. MEDLINE, Embase and Web of Science will be searched from 1 March 2020 to 31 August 2021. Following the title and abstract screening, we will identify, systematise and synthesise the available knowledge. Based on pilot searches, we preview three themes for descriptive evidence synthesis. The first theme relates to implementation and use of mHealth telemonitoring, including reported barriers. The second theme covers the interactions of the telemonitoring team within and between different levels of the healthcare system. The third theme addresses how this telemonitoring warrants the continuity of care, also during disease transition into deterioration or postacute sequelae. Ethics and dissemination The studied evidence is in the public domain, therefore, no specific ethics approval is required. Evidence dissemination will be via peer-reviewed publications, conference presentations and reports to the policy makers.

\section{INTRODUCTION}

The pandemic of COVID-19 is still ongoing and continues to impact various aspects of our lives. Until sufficient collective immunity will be achieved by mass vaccination, acute COVID-19 cases are expected to flourish.

\section{Strength and limitations of this study}

- The strength of this scoping review is in its comprehensive approach, designed to cover three major electronic databases in three languages over a period of 18 months.

- Another strength lies in rigorous implementation of the newest methodological guidelines for scoping reviews, as well as in utilisation of innovative approaches, such as automated text mining.

- Stakeholders representing different levels of the healthcare system and a range of medical subspecialties contributed to conceptualising of this scoping review.

- Due to a rapidly growing body of publications on COVID-19, our scoping review may inadvertently miss some publications that have not been assigned the used search tags and/or keywords.

- This scoping review focuses on the evidence synthesis related to acute COVID-19 and transition into postacute sequelae, whereas long COVID-19 will not be addressed in depth.

Addressing this challenge, many medical innovations are being implemented. ${ }^{1}$ They often rely on utilisation of information and communication technologies, thereby falling under the definition of eHealth technologies. $^{2}$ A particular subtype of eHealth is called mHealth. ${ }^{23}$ The latter concept enables assessments of health data, typically via a smartphone or tablet, and in conjunction with dedicated mobile apps. In addition, mHealth telemonitoring can employ specialised digital sensors capable of remote transmission of patient's data.

During the pandemic, the eHealth/ mHealth technologies emerged as popular tools for remote consultations of nonCOVID-19 conditions. ${ }^{4-10}$ mHealth technologies are also suitable for procuring the COVID-19 related health data. ${ }^{11-14}$ In this 
regard, mHealth technologies exhibit excellent scalability, which ranges from gathering population-level data $^{1516}$ to following, in real time, the symptoms of an individual patient.

The latter feature of mHealth telemonitoring is attractive, as it enables remote patient monitoring and remote patient stratification. ${ }^{17}$ Moreover, patient monitoring can be individualised to respect the patient's demographic and medical profile. Thereby, individual risks for severe COVID-19 and associated mortality, which both can be high in certain patient groups, ${ }^{18-21}$ can be given appropriate attention. Conversely, monitoring of acute COVID-19 could reveal signs of delayed convalescence and potential transition into postacute sequelae. The latter phenomenon has been attracting attention recently. ${ }^{22}$

While seemingly straightforward, implementation of mHealth technologies for monitoring of acute COVID-19 can be hindered by various issues. These issues include reluctant embrace by the end users, insufficient local expertise and resources for implementation, lacking qualifications by physicians and other healthcare professionals, and cybersafety concerns. Furthermore, this implementation could exhibit varying successes when applied to patients with differing affinity to digital media. Another difficulty could evolve when different levels of the healthcare system need to be integrated into one mHealth telemonitoring concept. For instance, deterioration of acute COVID-19 can occur very rapidly, necessitating prompt medical interventions. In contrast to a hospital setting, with its established standard operating procedures and proximity of a multidisciplinary response team, prompt responsiveness to deterioration of outpatient COVID-19 may not be trivial. This could partially negate the benefit of real-time monitoring.

The aforementioned issues provide a rationale for synthesis and dissemination of the evidence on successful mHealth telemonitoring of COVID-19. This is especially true for concepts involving horizontal and vertical integration across the healthcare system. It has been a year and a half since the COVID-19 pandemic had officially been announced, ${ }^{23}$ and a vast body of literature has been accumulated. Yet, based on our pilot literature sample, such systematic evidence synthesis is still lacking. In particular, the existing review articles either focus on remote medical services for non COVID-19 conditions (eg, refs 24-32) or do not sufficiently elaborate on the implementation concepts. ${ }^{15}$ 33-35 Moreover, to the best of our knowledge, structured literature synthesis has not been done with respect to the composition of the telemonitoring team. Similarly, the evidence synthesis is scarce on integration of different levels of the healthcare system implicated in the telemonitoring process. In addition, the data on postacute COVID-19 sequelae have only recently started to be compiled. Therefore, knowledge synthesis on the role of mHealth technologies in recognition of these sequelae is still lacking.

This rationale prompted us to plan a scoping review on the aforementioned subject. Our research question was conceptualised broad: 'What are the determinants of successful implementation and use of mHealth telemonitoring in acute outpatient COVID-19?'. The following text presents the scoping review protocol that will address this research question.

\section{METHODS AND ANALYSIS}

\section{Aims, objectives and methodological framework}

This scoping review aims to identify, systematise and synthesise the available knowledge on the implementation and use of mHealth telemonitoring in acute outpatient COVID-19. This overarching aim will entail three specific objectives. The first objective will be to provide a qualitative and categorised overview of implementation and execution of this telemonitoring. Our second objective will be to aggregate the published evidence on the composition of the telemonitoring team. By the third objective, we will describe the use of mHealth technology to warrant the continuity of care of outpatient COVID-19 in the context of recognition of acute deterioration or postacute sequelae.

This scoping review will follow the essential stages of the methodological framework for scoping reviews, based on the previously published guidelines, including the Preferred Reporting Items for Systematic Reviews and Meta-Analyses recommendation for Scoping Reviews (PRISMA-ScR; online supplemental file 1 for PRISMA-ScR Checklist and refs 36-41). In particular, this scoping review corresponds to the type 3 (summary and dissemination of research findings) and type 4 (identification of knowledge gaps) of the scoping reviews. ${ }^{36}$

\section{Search strategy and knowledge databases}

The search strategy aims for high coverage and sensitivity (recall). It was developed and test-run by the lead author (ND) and the chief librarian of the Central Medical Library of Philipps University of Marburg (SW) (online supplemental file 2) in discussions with team members.

The search strategy followed the PICO (Population, Intervention, Comparator, Outcome) criterion, ${ }^{42}$ with 'population' being acute COVID-19 (online supplemental file 2). In particular, we will consider publications on outpatients comprising: (A) asymptomatic adults (age $>18$ years old), typically identified via a PCR test and positive for SARS-CoV-2 and (B) symptomatic adults (age $>18$ years old) with clinical manifestations of COVID-19 (with or without positive PCR test). The 'intervention' will be mHealth home telemonitoring (online supplemental file 2 ). The latter was spelled out as different definitions of mHealth technology and the associated digital sensors (online supplemental file 2). The 'comparator' could be any (eg, standard practices of patient monitoring (such as by a phone call), monitoring without the use of portable digital devices) or none, and thus was not defined specifically in the search strategy.

With regard to the planned 'outcome', the following can be stated. Our pilot search identified a relative scarcity 
of publications on telemonitoring of acute COVID-19 as opposed to reports on telemedicine for non COVID-19 conditions. Furthermore, the identified pertinent reports present evidence on heterogenous topics. Addressing these two points, we formulated the 'outcome' as a combination of several potential end products of published studies.

The outcome no. 1 will concern the reports on implementation and use of mHealth telemonitoring (online supplemental file 2). These reports could present a general concept (eg, telemonitoring implemented in primary care only, or with integration with secondary and/or tertiary healthcare facilities), description of barriers to implementation or lists of the employed mHealth technology, evaluated endpoints and achieved successes.

The outcome no. 2 will aggregate the evidence on the composition and competencies of the telemonitoring team. The latter may comprise general practitioners, nurse practitioners, physician assistants, respirologists, intensive care specialists, information technology specialists or other experts. In the MEDLINE (online supplemental file 2) and other database searches, this 'Outcome' will not be specified separately, as the evidence on the team composition may be embedded in the description of the implementation process (eg, refs 16 17).

The outcome no. 3 will comprise all medical decisions related to changes of COVID-19 progression, either towards acute deterioration or postacute sequelae (online supplemental file 2). These decisions may comprise triage, hospitalisation, intensive care or other urgent measures, or may be those addressing the burgeoning postacute sequelae (online supplemental file 2).

Finally, the outcome no. 4 will be related to patients' adherence to medical procedures (online supplemental file 2).

The search strategy was developed principally using MEDLINE terms (Medical Subject Headings and Supplementary Concepts), enhanced by custom keywords (online supplemental file 2). The custom keywords were derived from the titles, abstracts or author-tagged keywords of the publications identified in the pilot run of the test strategy. These keywords were compiled manually, as well as by text mining algorithms. Text mining was conducted using the packages 'revtools' ${ }^{43}$ and 'litsearchr ${ }^{44}$ for R statistical environment. ${ }^{45}$ Addition of custom keywords to the search strategy aimed to increase the sensitivity of the latter.

To warrant sufficient coverage, we will search the following electronic databases: Ovid/MEDLINE, Ovid/ Embase and Web of Science. The combination of these three databases is expected to yield the median sensitivity of the literature search of over $97 \%{ }^{46}$ The search terminology for the latter two databases will be adapted from the one developed for MEDLINE (online supplemental file 2). The search languages will be English (American and British spelling), German and French. The publication timeframe is set from 1 March 2020 to 31 August 2021. The start date was selected as to include the first date of the month when the COVID-19 pandemic had been announced. ${ }^{23}$ The end date marks the date of compilation of the final version of the study protocol. The actual study (evidence screening and synthesis) is planned between the start date of 1 October 2021 and the end date of 15 November 2021.

To further increase the coverage, we will use Cochrane Library and ClinicalTrial.gov. The latter two databases will inform the secondary searches, as described further.

\section{Eligibility criteria for publications on mHealth telemonitoring}

As a general inclusion criterion, we will consider original publications that describe mHealth home telemonitoring of patients with acute COVID-19. This telemonitoring is supposed to generate a stream of real-time electronic signals from one or several digital sensor(s), with or without integration by a mobile app. The primary use of these signals should be for the objective and repeated remote assessment of patient's COVID-19 condition by a telemonitoring team. All types of original publications (observational and experimental; cross-sectional or longitudinal; RCT or non-randomised or non-controlled trial, case series or case reports) will be included for screening.

Excluded from the screening will be original publications on mHealth home telemonitoring if such telemonitoring was used as a substitute for personal visits to the clinic of patients with non COVID-19 conditions (eg, diabetes, Chronic Obstructive Pulmonary Disease (COPD), cardiovascular disease, etc). Conference abstracts and proceedings will also be excluded, as will the editorials, commentaries, letters to editor, essays, book chapters, books and contents of internet websites.

Protocols for clinical trials (ClinicalTrial.gov and clinical trial protocols in MEDLINE) will not be included per se but will inform the secondary literature search in MEDLINE for publications that present the results of these clinical trials. Similarly, relevant review publications (such as those identified in the Cochrane Library) will not be included but will inform the secondary search in MEDLINE. Specifically, the references of review publications will be screened for potential suitability in this scoping study ('forward snowballing'). We will also identify original publications which cite these review publications ('reverse snowballing').

Before screening for inclusion in the final reference sample, the references will first undergo deduplication. To this end, the references identified through all searches will be subjected to automated deduplication (the aforementioned package 'revtools ${ }^{43}$ ) and comprehensive manual deduplication in Endnote, as per published optimised protocol. ${ }^{47}$

\section{Screening for suitable publications}

Following deduplication, the publications will undergo manual screening. The latter will be conducted as follows. First, the titles and abstracts will be screened by the reviewers' team comprising the lead author and two other reviewers (SH and $\mathrm{LN})$. We will use the online 
software Rayyan ${ }^{48}$ to enable independent and collaborative screening. The Cohen's kappa coefficient will be used to quantitatively assess the inter-reviewer reproducibility of title and abstract screening. The expectation is that this coefficient will be at least 0.8 . Potential disagreements between these reviewers will be solved on consultation with the fourth member of reviewers' team (SK).

Following the previous screening step, full-text publications will be procured using publications' digital object identifiers (doi's) or manually.

\section{Charting the data}

The full-text screening will be done by the lead author, supported by other members of the reviewers' team $(\mathrm{SH}$, LN and SK) and will yield a final reference sample for descriptive synthetic analysis. If needed, consultations will be held with other coauthors.

The information from the final reference sample will be compiled in the data extraction table, which was developed and tested during the pilot searches. This table includes the publication ID (authors, title, journal and doi), country of study's origin, research question/hypothesis/objectives of the study, type of the study (RCT, nonrandomised or non-controlled trial, observational study, case series or case report), study design (cross-sectional or longitudinal), population (patients' age, race and ethnicity, sex and gender, comorbidities, stage of the disease (asymptomatic SARS-CoV-2 carriage/oligosymptomatic or acute COVID-19), detailed description of telemonitoring (ie, which mHealth device(s) was/were used, particularities of the use), duration of digital telemonitoring, availability and characteristics of the comparator. Publications' outcomes and conclusions will also be documented.

In addition to demographic and clinical information, we will compile the details of processing of the incoming status feeds, such as the thresholds on which the monitoring medical team was alerted, the protocols of subsequent responses by the medical team and the reported medical interventions (eg, phone call, text message with referral to emergency department, etc). If available, we will gather information on the composition and competencies of the telemonitoring team. We will also record the information on whether and how the included studies addressed patients' adherence to telemonitoring. Processing of potential measurement errors (either patient-associated errors or due to intrinsic variability of mHealth devices) will also be noted.

\section{Evidence synthesis}

Descriptive synthesis of the retrieved evidence will be conducted, provided that there will be at least two identified relevant publications in the final reference sample. On finding of only one pertinent publication, we will use it in the scoping review as an example of the mHealth telemonitoring concept. To ensure the bias-free evidence synthesis, $10 \%$ of publications in the final reference sample will be randomly selected and subjected to independent review by the second reviewer $(\mathrm{SH})$. If the number of publications will be low, and allocation of $10 \%$ not feasible, a different proportion of the publications in the reference sample (eg, half of the publications in case of the reference sample comprising $<10$ publications) will be selected for the independent review by the second reviewer. Finally, the retrieved data and descriptive synthesis will jointly be discussed within the reviewers' team, as well as with other coauthors. The joint authors' team include the end users of mHealth technologies at primary, secondary and tertiary levels of the healthcare system.

The evidence from retrieved and selected publications will be presented in a structured form as a table, with rows presenting individual publications and columns presenting the key publication variables (eg, study's research question, country of origin, study design and population, type of mHealth technology used and other pertinent details). We will also describe the synthesised evidence in the text form. The text description will permit logical organisation of the evidence based on the three specific objectives of this scoping review.

As per these objectives, we preview three themes by which we will carry out the evidence synthesis. First, we will describe the process of implementation and use of mHealth home telemonitoring. In particular, we are interested to learn, aggregate and disseminate the knowledge about potential common barriers to implementation of mHealth technology (eg, renumeration issues, cybersafety aspects, costs of pertinent technology and lack of local expertise) that had been encountered and overcome by the authors of previous publications. Second, we will aggregate the published knowledge on the composition of the telemonitoring team. In many instances, this telemonitoring may invoke interactions within and between different levels of the healthcare system. Thereby, it is essential to synthesise the knowledge as to whether the telemonitoring team, from the onset on, should be multidisciplinary, with affiliations with the secondary/tertiary levels of the healthcare system. Alternatively, such team could represent only primary care experts but be backed up by a support (outreach) team comprising the secondary/tertiary level specialists. Third, we will aggregate the knowledge on the standard operating procedures that warrant the continuity of care in the home-telemonitored patients, such as by addressing changes in progression of COVID19 , either towards acute deterioration or postacute sequelae.

This review does not plan to critically appraise the available published evidence. Such appraisal (eg, of the reported successes of digital home telemonitoring in comparison to standard care) will be resorted to a subsequent systematic review. The decision as to whether to carry out a systematic review will be made depending on the number and quality of the identified references. 


\section{Patient and public involvement}

As mentioned previously, the joint authors' team includes the medical end users of mHealth telemonitoring at different levels of the healthcare system. Thereby, the development of this protocol benefited from regular discussions within the team. No patient or public involvement took place in the development of this scoping review protocol. This scoping review may identify the need for a subsequent systematic review. At that stage, medical end users of these technologies will again be involved. We will also seek the patients' reflection and perspective on mHealth home monitoring. To this end, we, at the stage of drafting a subsequent systematic review, will consult former patients with COVID-19, especially those representing vulnerable groups (eg, refugees, those with a handicap, patients in advanced age or residents of longterm care facilities).

\section{DISCUSSION}

Home telemonitoring is an attractive solution in the context of the still ongoing pandemic and its extensive burden on the tertiary healthcare system. The majority of patients with COVID-19 can and do endure the acute disease in an outpatient setting. ${ }^{49-53}$ Addressing this fact, various solutions have been proposed for monitoring of non-hospitalised patients with acute COVID-19. These solutions range from online questionnaires ${ }^{16}$ to teleconsultations or videoconsultations. ${ }^{54}$ Disease deterioration can, however, develop rapidly in COVID-19. Digital sensors, especially pulse oximetry, are helpful as harbingers of such deterioration. ${ }^{1852535556}$ In this regard, mHealth-based home telemonitoring, comprising pulse oximetry and other pertinent wearables, represents a crucial bridge between COVID-19 deterioration and a subsequent medical response.

In a similar manner, the use of mHealth wearables (eg, fitness trackers, portable spirometers and monitors for cardiovascular function) may be helpful for early recognition and subsequent monitoring of postacute COVID-19 sequelae. As of the past few months, there is a growing attention to those sequelae (also known as 'long COVID-19' or 'chronic COVID-19'. ${ }^{22}$ 57-59 The mHealth technologies that monitor functional status of the cardiopulmonary system are particularly well suited for timely recognition of troubled physical recovery. This is especially important, given the current lack of individual predictors of these sequelae. ${ }^{60}$

As mentioned previously, this attractive concept may be hindered by various factors, including insufficient embrace by the end users, unresolved issues with fees and renumeration, as well as by technological and legal problems. For example, pulse oximetry can generate substantial measurement artefacts. ${ }^{56}$ This necessitates appropriate guidance and counselling of the patient, especially to warrant the conformity with the measurement standards. ${ }^{61}$ In addition, implementation of mHealth technology can be viewed as disruptive by the medical personnel ${ }^{62}$ who are already overworked during the pandemic and may lack sufficient expertise in this area. At present, the majority of practising health professionals are not adequately trained in utilisation of mHealth and telemedicine. Thereby, additional efforts are needed to integrate these concepts into daily clinical practice.

On the patient level, too, there could exist certain barriers. In particular, proficiency with digital devices, affinity to digital media and acceptability of digital healthcare may differ substantially among patients, depending on their age, ${ }^{6364}$ education level $^{65}$ or minority representation (eg, recent immigrants, ${ }^{64}$ refugees $^{66}$ or religious minority communities). ${ }^{66}$ On top of this, adherence to telemonitoring routines may vary, depending on the severity and duration of the disease,${ }^{67}$ or duration ${ }^{68}$ and frequency ${ }^{69}$ of telemonitoring events.

Therefore, identification and dissemination of successful concepts on digital home telemonitoring of COVID-19 using mHealth technology is crucial. The present scoping review will focus on this goal. In particular, this review will structure and synthesise the available evidence on implementation and use of mHealth home telemonitoring in acute COVID-19. Furthermore, this review will aggregate the knowledge on prerequisites, both in personnel and technology, that are essential for continuity of care and seamless integration of this telemonitoring with different levels of the healthcare system.

In conclusion, prompt aggregation and synthesis of the evidence on implementation and use of mHealth technology in acute outpatient COVID-19 is an exigency. This scoping review is the necessary step towards future critical evidence appraisal in this area.

\section{ETHICS AND DISSEMINATION}

For proper dissemination of the evidence synthesis, we plan to publish this scoping review in a peer-reviewed journal, as well as share the findings at medical conferences, both on primary care (eg, German Society of General Practice and Family Medicine; North American Primary Research Group) and respiratory medicine (American Thoracic Society, European Respiratory Society and German Society of Pulmonary Medicine). In addition, we will prepare a concept document that will be submitted to policy makers in Germany. Our group works as part of the national consortium of university hospitals (egePAN Unimed). This consortium developed a website (www.egepan.de) that could be used for podcasts and other tools for dissemination of the synthesised evidence.

As mentioned previously, this scoping review may inform the authors' team about the feasibility of a systematic review in this area. Such systematic review could be done in direct collaboration with the national and European institutes specialising in advancing evidence-based digital medicine. 


\section{Author affiliations}

${ }^{1}$ Department of Internal, Respiratory and Critical Care Medicine, Philipps-Universitat Marburg, Marburg, Hessen, Germany

${ }^{2}$ Department of Pediatrics, McGill University Faculty of Medicine and Health Sciences, Montreal, Québec, Canada

${ }^{3}$ Department of Digital Medicine, Bielefeld University Faculty of Medicine, Bielefeld, Germany

${ }^{4}$ Institute of General Practice, Goethe University Frankfurt, Frankfurt am Main, Hessen, Germany

${ }^{5}$ Central Medical Library, Philipps-Universitat Marburg, Marburg, Hessen, Germany ${ }^{6}$ Department of Pneumology and Intensive Care Medicine, University Hospital Aachen, Aachen, Nordrhein-Westfalen, Germany

${ }^{7}$ Department of Respiratory Medicine, Hospital of the Goethe University Frankfurt, Frankfurt am Main, Hessen, Germany

Contributors ND conceived this study, conducted the pilot searches and drafted the manuscript. ND and SW conceptualised and test run the search protocol. SK, $\mathrm{SH}, \mathrm{LN}, \mathrm{BSM}$ and CB contributed to the development of the study protocol and formulation of the research question. SK, BSM, MD, GGUR and CV critically revised and contributed to the manuscript draft. All authors approved the final version of the manuscript. ND is accountable for all aspects of the work in ensuring that questions related to the accuracy or integrity of any part of the work are appropriately investigated and resolved.

Funding This study protocol was supported by internal funds of Department of Internal, Respiratory and Critical Care Medicine (Philipps-University of Marburg, Marburg, Germany), German Centre for Lung Research (DZL) and The Federal Ministry of Education and Research (BMBF; egePAN Unimed Project, award number 01kX2021)

\section{Competing interests None declared.}

Patient consent for publication Not applicable.

Provenance and peer review Not commissioned; externally peer reviewed.

Supplemental material This content has been supplied by the author(s). It has not been vetted by BMJ Publishing Group Limited (BMJ) and may not have been peer-reviewed. Any opinions or recommendations discussed are solely those of the author(s) and are not endorsed by BMJ. BMJ disclaims all liability and responsibility arising from any reliance placed on the content. Where the content includes any translated material, BMJ does not warrant the accuracy and reliability of the translations (including but not limited to local regulations, clinical guidelines, terminology, drug names and drug dosages), and is not responsible for any error and/or omissions arising from translation and adaptation or otherwise.

Open access This is an open access article distributed in accordance with the Creative Commons Attribution Non Commercial (CC BY-NC 4.0) license, which permits others to distribute, remix, adapt, build upon this work non-commercially, and license their derivative works on different terms, provided the original work is properly cited, appropriate credit is given, any changes made indicated, and the use is non-commercial. See: http://creativecommons.org/licenses/by-nc/4.0/.

\section{ORCID iDs}

Nurlan Dauletbaev http://orcid.org/0000-0002-7114-1041

Beate S Müller http://orcid.org/0000-0002-6745-1047

\section{REFERENCES}

1 Golinelli D, Boetto E, Carullo G, et al. Adoption of digital technologies in health care during the COVID-19 pandemic: systematic review of early scientific literature. J Med Internet Res 2020;22:e22280.

2 Saner $\mathrm{H}$, van der Velde $\mathrm{E}$. eHealth in cardiovascular medicine: a clinical update. Eur J Prev Cardiol 2016;23:5-12.

3 Burke LE, Ma J, Azar KMJ, et al. Current science on consumer use of mobile health for cardiovascular disease prevention: a scientific statement from the American heart association. Circulation 2015;132:1157-213.

4 Begg A. Diabetes virtual clinics: technology-enabled care services (TECS) - the new norm? Pract Diab 2020;37:188-90. doi:10.1002/ pdi.2299

5 Levine $\mathrm{OH}$, McGillion M, Levine M. Virtual cancer care during the COVID-19 pandemic and beyond: a call for evaluation. JMIR Cancer 2020;6:e24222. doi:10.2196/24222

6 Rammo R, Gostkowski M, Rasmussen PA, et al. The need for digital health solutions in deep brain stimulation for Parkinson's disease in the time of COVID-19 and beyond. Neuromodulation 2021;24:331-6.
7 Wootton SL, King M, Alison JA, et al. COVID -19 rehabilitation delivered via a telehealth pulmonary rehabilitation model: a case series. Respirol Case Rep 2020;8:6. doi:10.1002/rcr2.669

8 Sasangohar F, Bradshaw MR, Carlson MM, et al. Adapting an outpatient psychiatric clinic to telehealth during the COVID-19 pandemic: a practice perspective. J Med Internet Res 2020;22:e22523.

9 Quinn LM, Davies MJ, Hadjiconstantinou M. Virtual consultations and the role of technology during the COVID-19 pandemic for people with type 2 diabetes: the UK perspective. J Med Internet Res 2020;22:e21609.

10 Esper GJ, Sweeney RL, Winchell E, et al. Rapid systemwide implementation of outpatient telehealth in response to the COVID-19 pandemic. J Healthc Manag 2020;65:443-52.

11 Silven AV, Petrus AHJ, Villalobos-Quesada M, et al. Telemonitoring for patients with COVID-19: recommendations for design and implementation. J Med Internet Res 2020;22:e20953.

12 Wong CK, Ho DTY, Tam AR, et al. Artificial intelligence mobile health platform for early detection of COVID-19 in quarantine subjects using a wearable biosensor: protocol for a randomised controlled trial. BMJ Open 2020;10:e038555

13 Un K-C, Wong C-K, Lau Y-M, et al. Observational study on wearable biosensors and machine learning-based remote monitoring of COVID-19 patients. Sci Rep 2021:11:4388.

$14 \mathrm{Xu} \mathrm{H}$, Huang S, Qiu C, et al. Monitoring and management of home-quarantined patients with COVID-19 using a WeChat-based telemedicine system: retrospective cohort study. J Med Internet Res 2020;22:e19514.

15 Gunasekeran DV, Tseng RMWW, Tham Y-C, et al. Applications of digital health for public health responses to COVID-19: a systematic scoping review of artificial intelligence, telehealth and related technologies. NPJ Digit Med 2021;4:40.

16 Yordanov Y, Dechartres A, Lescure X, et al. Covidom, a telesurveillance solution for home monitoring patients with COVID-19. J Med Internet Res 2020;22:e20748.

17 Morgan AU, Balachandran M, Do D. Remote monitoring of patients with Covid-19: design, implementation, and outcomes of the first 3,000 patients in COVID Watch. NEJM Catalyst 2020;21:1-12.

18 Cheng A, Caruso D, McDougall C. Outpatient management of COVID-19: rapid evidence review. Am Fam Physician 2020;102:478-86.

19 Docherty AB, Harrison EM, Green CA, et al. Features of 20133 UK patients in hospital with covid-19 using the ISARIC WHO Clinical Characterisation Protocol: prospective observational cohort study. BMJ 2020;369:m1985.

20 Richardson S, Hirsch JS, Narasimhan M, et al. Presenting characteristics, comorbidities, and outcomes among 5700 patients hospitalized with COVID-19 in the new York City area. JAMA 2020;323:2052-9.

21 Long L, Zeng X, Zhang X, et al. Short-term outcomes of COVID-19 and risk factors for progression. Eur Respir J 2020;55. doi:10.1183/13993003.00990-2020. [Epub ahead of print: 27 May 2020].

22 Sudre CH, Murray B, Varsavsky T. Attributes and predictors of long COVID. Nat Med 2021.

23 WHO. WHO Director-General's opening remarks at the media briefing on COVID-19, 2020.

24 Novara G, Checcucci E, Crestani A, et al. Telehealth in urology: a systematic review of the literature. how much can telemedicine be useful during and after the COVID-19 pandemic? Eur Urol 2020;78:786-811.

25 Shorey S, Lau LST, Tan JX, et al. Families with children with neurodevelopmental disorders during COVID-19: a scoping review. $J$ Pediatr Psychol 2021. doi:10.1093/jpepsy/jsab029. [Epub ahead of print: 24 Mar 2021].

26 Venketasubramanian N, Anderson C, Ay H, et al. Stroke care during the COVID-19 pandemic: international expert panel review. Cerebrovasc Dis 2021:50:1-17.

27 Pooni R, Pageler NM, Sandborg C, et al. Pediatric subspecialty telemedicine use from the patient and provider perspective. Pediatr Res 2021:1-6.

28 Tham Y-C, Husain R, Teo KYC, et al. New digital models of care in ophthalmology, during and beyond the COVID-19 pandemic. Br J Ophthalmol 2021. doi:10.1136/bjophthalmol-2020-317683. [Epub ahead of print: 22 Mar 2021].

29 Gosse PJ, Kassardjian CD, Masellis M, et al. Virtual care for patients with Alzheimer disease and related dementias during the COVID-19 era and beyond. CMAJ 2021;193:E371-7.

30 Doraiswamy S, Jithesh A, Mamtani R, et al. Telehealth use in geriatrics care during the COVID-19 Pandemic-A scoping review and evidence synthesis. Int J Environ Res Public Health 2021;18. doi:10.3390/ijerph18041755. [Epub ahead of print: 11 Feb 2021]. 
31 Su Z, McDonnell D, Liang B, et al. Technology-based health solutions for cancer caregivers to better shoulder the impact of COVID-19: a systematic review protocol. Syst Rev 2021;10:43.

32 Hubert GJ, Corea F, Schlachetzki F. The role of telemedicine in acute stroke treatment in times of pandemic. Curr Opin Neurol 2021;34:22-6.

33 Doraiswamy S, Abraham A, Mamtani R, et al. Use of telehealth during the COVID-19 pandemic: Scoping review. J Med Internet Res 2020;22:e24087.

34 Monaghesh E, Hajizadeh A. The role of telehealth during COVID-19 outbreak: a systematic review based on current evidence. BMC Public Health 2020;20:1193.

35 Ding X, Clifton D, Ji N, et al. Wearable sensing and telehealth technology with potential applications in the coronavirus pandemic. IEEE Rev Biomed Eng 2021;14:48-70.

36 Arksey H, O'Malley L. Scoping studies: towards a methodological framework. Int J Soc Res Methodol 2005;8:19-32.

37 Colquhoun HL, Levac D, O'Brien KK, et al. Scoping reviews: time for clarity in definition, methods, and reporting. J Clin Epidemiol 2014;67:1291-4.

38 Levac D, Colquhoun H, O'Brien KK. Scoping studies: advancing the methodology. Implement Sci 2010;5:69.

39 Lockwood C, Tricco AC. Preparing scoping reviews for publication using methodological guides and reporting standards. Nurs Health Sci 2020;22:1-4.

40 Tricco AC, Lillie E, Zarin W, et al. PRISMA extension for scoping reviews (PRISMA-ScR): checklist and explanation. Ann Intern Med 2018;169:467-73.

41 Peters MDJ, Godfrey CM, Khalil H, et al. Guidance for conducting systematic scoping reviews. Int J Evid Based Healthc 2015;13:141-6.

42 Richardson WS, Wilson MC, Nishikawa J, et al. The well-built clinical question: a key to evidence-based decisions. ACP J Club 1995;123:A12-13.

43 Westgate MJ. revtools: an R package to support article screening for evidence synthesis. Res Synth Methods 2019;10:606-14.

44 Grames EM, Stillman AN, Tingley MW. An automated approach to identifying search terms for systematic reviews using keyword cooccurrence networks. Methods Ecol Evol 2019;10:1645-54.

45 R Core Team. R: a language and environment for statistical computing. $\mathrm{R}$ foundation for statistical computing, Vienna, Austria, 2020. Available: http://www.r-project.org/index.html

46 Bramer WM, Rethlefsen ML, Kleijnen J, et al. Optimal database combinations for literature searches in systematic reviews: a prospective exploratory study. Syst Rev 2017;6:245.

47 Bramer WM, Giustini D, de Jonge GB, et al. De-duplication of database search results for systematic reviews in endnote. J Med Libr Assoc 2016;104:240-3.

48 Ouzzani M, Hammady H, Fedorowicz Z, et al. Rayyan-a web and mobile APP for systematic reviews. Syst Rev 2016;5:210.

49 Preskorn $\mathrm{SH}$. The $5 \%$ of the population at high risk for severe COVID-19 infection is identifiable and needs to be taken into account when reopening the economy. J Psychiatr Pract 2020;26:219-27.

50 Hamer M, Gale CR, Kivimäki M, et al. Overweight, obesity, and risk of hospitalization for COVID-19: a community-based cohort study of adults in the United Kingdom. Proc Natl Acad Sci U S A 2020;117:21011-3.

51 Reilev M, Kristensen KB, Pottegård A, et al. Characteristics and predictors of hospitalization and death in the first 11122 cases with a positive RT-PCR test for SARS-CoV-2 in Denmark: a nationwide cohort. Int J Epidemiol 2020;49:1468-81.
52 Sun $\mathrm{H}$, Jain $\mathrm{A}$, Leone MJ, et al. CoVA: an acuity score for outpatient screening that predicts coronavirus disease 2019 prognosis. $J$ Infect Dis 2021;223:38-46.

53 Blair PW, Brown DM, Jang M, et al. The clinical course of COVID-19 in the outpatient setting: a prospective cohort study. Open Forum Infect Dis 2021;8:ofab007.

54 Krenitsky NM, Spiegelman J, Sutton D, et al. Primed for a pandemic: implementation of telehealth outpatient monitoring for women with mild COVID-19. Semin Perinatol 2020;44:151285.

55 Wilkerson RG, Adler JD, Shah NG, et al. Silent hypoxia: a harbinger of clinical deterioration in patients with COVID-19. Am J Emerg Med 2020;38:2243.e5-2243.e6.

56 Luks AM, Swenson ER. Pulse oximetry for monitoring patients with COVID-19 at home. potential pitfalls and practical guidance. Ann Am Thorac Soc 2020;17:1040-6.

57 Mandal S, Barnett J, Brill SE, et al. 'Long-COVID': a crosssectional study of persisting symptoms, biomarker and imaging abnormalities following hospitalisation for COVID-19. Thorax 2021;76:396-8.

58 Baig AM. Deleterious outcomes in long-hauler COVID-19: the effects of SARS-CoV-2 on the CNS in chronic COVID syndrome. ACS Chem Neurosci 2020;11:4017-20.

59 Nalbandian A, Sehgal K, Gupta A, et al. Post-acute COVID-19 syndrome. Nat Med 2021;27:601-15.

60 Moreno-Pérez O, Merino E, Leon-Ramirez J-M, et al. Post-acute COVID-19 syndrome. incidence and risk factors: a Mediterranean cohort study. J Infect 2021;82:378-83.

61 Philip KEJ, Bennett B, Fuller S, et al. Working accuracy of pulse oximetry in COVID-19 patients stepping down from intensive care: a clinical evaluation. BMJ Open Respir Res 2020;7:e000778.

62 Odendaal WA, Anstey Watkins J, Leon N, et al. Health workers' perceptions and experiences of using mHealth technologies to deliver primary healthcare services: a qualitative evidence synthesis. Cochrane Database Syst Rev 2020;3:Cd011942.

63 Seifert A, Batsis JA, Smith AC. Telemedicine in long-term care facilities during and beyond COVID-19: challenges caused by the digital divide. Front Public Health 2020;8:601595.

64 Badawy SM, Radovic A. Digital approaches to remote pediatric health care delivery during the COVID-19 pandemic: existing evidence and a call for further research. JMIR Pediatr Parent 2020;3:e20049.

65 Nguyen A, Mosadeghi S, Almario CV. Persistent digital divide in access to and use of the Internet as a resource for health information: results from a California population-based study. Int $J$ Med Inform 2017;103:49-54.

66 Fagherazzi G, Goetzinger C, Rashid MA, et al. Digital health strategies to fight COVID-19 worldwide: challenges, recommendations, and a call for papers. J Med Internet Res 2020;22:e19284.

67 Kerby TJ, Asche SE, Maciosek MV, et al. Adherence to blood pressure telemonitoring in a cluster-randomized clinical trial. $J$ Clin Hypertens 2012;14:668-74.

68 Ware P, Dorai M, Ross HJ, et al. Patient adherence to a mobile phone-based heart failure telemonitoring program: a longitudinal mixed-methods study. JMIR Mhealth Uhealth 2019;7:e13259.

69 Lang C, Voigt K, Neumann R, et al. Adherence and acceptance of a home-based telemonitoring application used by multimorbid patients aged 65 years and older. J Telemed Telecare 2020;1357633:x20901400 